\title{
The CosmoAmautas project for equitable scientific education in Peru
}

\author{
Equitable and high-quality scientific education is essential for fighting social inequalities and misinformation. \\ The CosmoAmautas project aims to address this need and use astronomy to contribute to an accessible and \\ decentralized scientific education in Peru.

\section{Gabriela Calistro Rivera, Daniella Bardalez Gagliuffi, Diego Alvarado Urrunaga, Lisseth Gonzales Quevedo, Daniel Kleffman, Erick Meza, Adita Quispe Quispe, Jenny Margot Ramos Lázaro, José Ricra, Bruno Rodríguez Marquina and Erika Torre Ramirez}

W e all live under the same sky. This accessibility places astronomy in a unique position to inspire awe and connect people from a variety of backgrounds. Astronomy thus has the potential to promote equity and diversity in society, while providing the technical basis for the inclusion of the scientific method in education. Peru is a country with an astronomical tradition that goes back millennia to the most ancient cultures in the Americas ${ }^{1}$. This heritage, in conjunction with the under-exploited world-class quality of the Peruvian skies ${ }^{2}$, makes astronomy a highly applicable tool for scientific and socioeconomic development, as defined by the United Nations Sustainable Development Goals ${ }^{3}$. Such a contribution is timely and urgent as countries in Latin America are experiencing the worst educational crisis in their history due to the COVID-19 pandemic ${ }^{4}$. The case of Peru has been particularly critical, with more than $85 \%$ of schools closed throughout 2020 and 2021 , and the public education system in a declared state of emergency until 2022. Even before the pandemic, Peru had one of the lowest scores in Latin America in the areas of science and maths based on Programme for International Student Assessment (PISA) evaluations ${ }^{5}$. In particular, the most striking gaps are found between rural and urban female students as evidenced by an illiteracy rate of $21.2 \%$ for the female rural population over 15 years old compared with the average national illiteracy rate of $5.5 \%$ (ref. ${ }^{6}$ ), reflecting severe inequalities in education.

\section{What is CosmoAmautas?}

'Amauta' means 'teacher' or 'knowledgeable person' in Quechua, the most widely spoken Indigenous language in Peru. CosmoAmautas was developed with the vision to contribute to a stronger and more equitable scientific education in the country, focusing on the most vulnerable socioeconomic sectors that are commonly distributed in rural regions away from the capital city of Lima. CosmoAmautas is an initiative of early-career Peruvian astronomers working abroad together with astronomers with affiliations spanning most local institutions and universities involved in astronomy in Peru. The collaboration between young members of a wide range of local institutions was an important goal accomplished by the programme, as a substantial long-term impact can only be achieved through a strong collaborative attitude, in particular in a small astronomical community.

To use astronomy as a tool for socioeconomic development, it is compulsory to develop astronomy locally. CosmoAmautas therefore adopts a multi-level strategy consisting of three tiers: (1) research at the university level, (2) outreach at the community level and (3) education at the high-school level. Although the high-school-education tier aims at promoting long-term scientific human capital, the university-level tier is developing astronomical research in the short term, which is currently incipient within physics departments in Peruvian universities. With this aim, six physics undergraduate students were selected in 2020 to participate in the mentoring and research programmes led by the more senior members of the team. In addition to developing their soft skills, such as leadership and science communication, the students led research programmes in areas such as stellar, extragalactic astronomy and cosmology. This training and contact with members of the international scientific community is crucial to increase the students' chances to access international graduate schools and, consequently, future careers in astrophysics. In the following years, we envisage building a stronger network of Peruvian astronomers abroad participating in research and mentoring of local students, as both research infrastructure and human capital is required to strengthen local astronomical research in the future. While the outreach events (second tier) in rural communities were postponed to minimize risks owing to the COVID-19 pandemic, the education tier evolved into a new educational model, on which we expand below.
A hands-on virtual education model The teacher training event took place virtually in six all-day sessions in April and May 2021. Fifty teachers were selected among 97 applicants, from three geographically diverse rural regions highlighted in Fig. 1: the coastal region of Moquegua, the Andean region of Junin and the rainforest Amazonian region of Loreto. In particular, the regions of Moquegua and Junin were chosen owing to their relevance as hosts of two of the main astronomical facilities in the country: a 1-m telescope at the Moquegua Astronomical Observatory (CONIDA) and a 0.51-m telescope (OAUNI) at the Huancayo Observatory (IGP). The six sessions of the teacher training event were divided into different topics across all cosmic scales, including: the Earth-Sun-Moon system, Solar System, stars, exoplanets, galaxies and cosmology. Each session (six hours) was divided into two main parts. The first half consisted of hands-on enquiry-based learning activities (Fig. 2) in which the teachers explored the topic in small groups with the support of an instructor, approaching one specific question, hypothesizing the answer, testing these hypotheses through experimentation and finally presenting their conclusions. These activities included the measurement of the Earth's diameter, the comparison of Solar System scales using rice grains, the exploration of stellar parameters using a home-made spectrograph, the simulated search for exoplanets from real data, the 


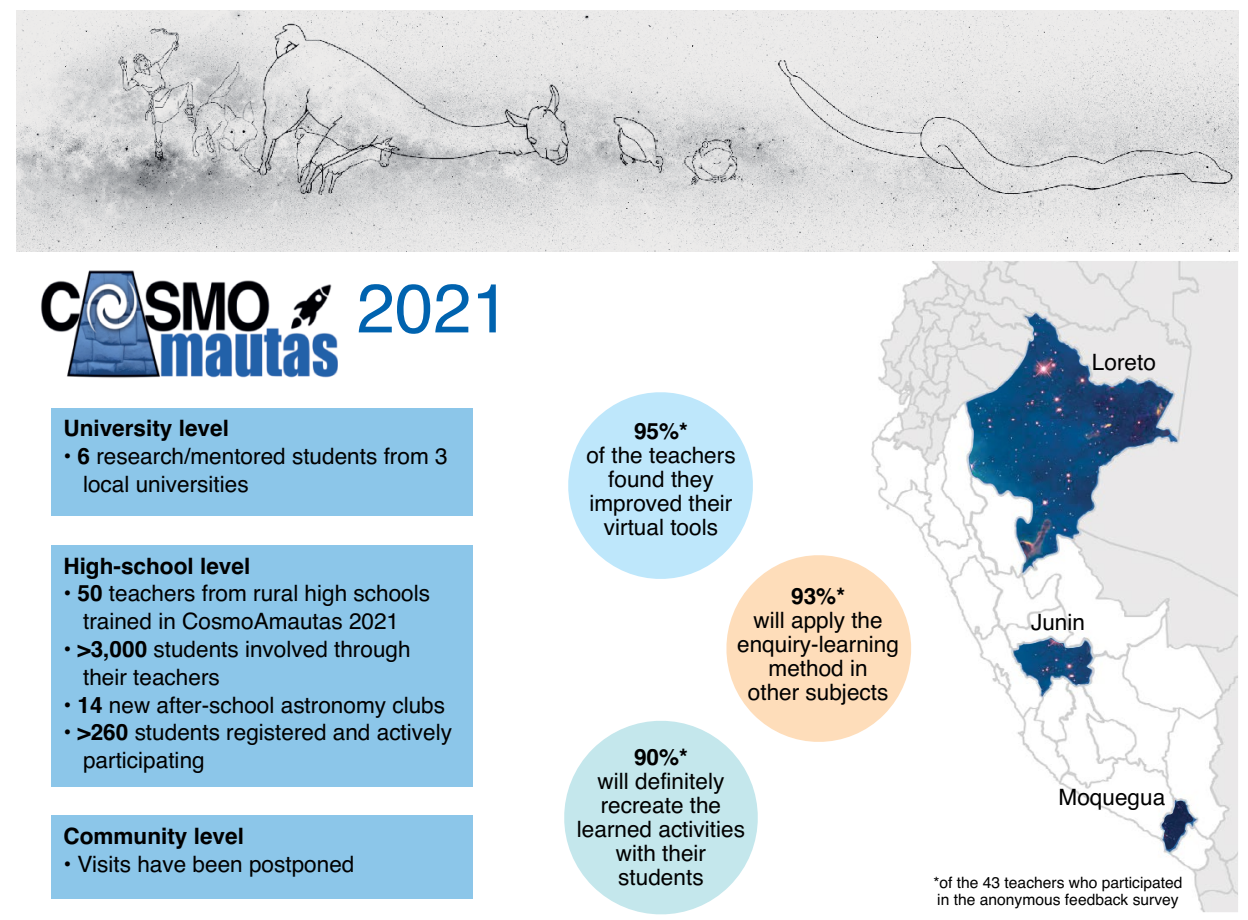

Fig. 1 | Overview of the CosmoAmautas multi-level strategy and outcomes. In the right panel, the Peruvian regions targeted in CosmoAmautas 2021 are shown. In the top panel, Inca dark constellations designed by members of our team (J. Ricra \& J. Maguiña) are drawn on a modified panoramic image of the Milky Way (ESO/S. Brunier).

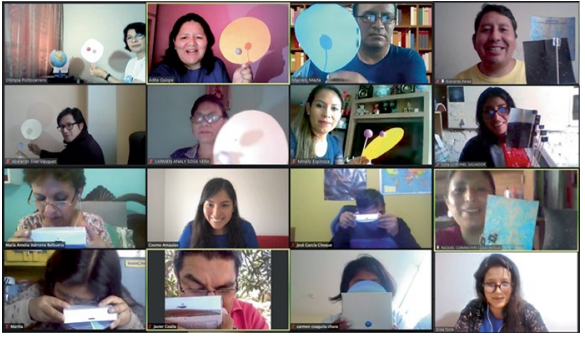

Fig. 2 | Enquiry-based learning activities in a virtual setting. High-school teachers and instructors carrying out hands-on activities on exoplanets, spectroscopy and the early Universe. Credit: Gabriela Calistro Rivera.

measurement of our Galaxy's rotation using GAIA data, and the reconstruction of cosmic history using the Hubble Ultra-Deep Field image. The second half of the day consisted of interactive lectures on astrophysics, science education and pedagogy, gender balance, local ancestral astronomy and climate change.

Two aspects were essential in defining the CosmoAmautas educational strategy: multiplicity and self-sustainability.

Our teacher training programme is multiplicative as it reaches a larger number of students through their teachers than student-only events could have done, with more than 3,000 students from rural high schools involved through 50 science and mathematics teachers that participated in the workshop. It is also self-sustainable, as the skills learnt by the teachers are also applicable for the next generations of students, and some of the most motivated teachers from our programme are training their local colleagues on their own initiative.

The COVID-19 pandemic required adapting our programme to a virtual format. This requirement had a few advantages as it enabled the targeting of three regions spanning a distance of $1,500 \mathrm{~km}$ across the country, as well as allowing the participation of teachers who would have been limited by their household responsibilities. Indeed, a few of the teachers (mostly female) attended the lectures accompanied by their children. The imposed virtual education system also revealed new necessities from the teachers in terms of virtual teaching strategies, which prompted us to redesign our hands-on enquiry-learning methodology for achieving the same impact in a virtual context. This change motivated the introduction of smaller groups of teachers using breakout rooms, and we developed inexpensive and innovative digital educational tools, such as astronomy-focused educational video games tailored to complement our activities (available in Spanish on our webpage www. cosmoamautas.org).
To ensure the equitable participation and engagement of teachers, we designed and shipped educational boxes across the country with all required materials to be used for the enquiry-based learning activities. The materials included a text and activity book, which our team wrote to respond to a lack of accessible yet up-to-date astronomy content in Spanish at an advanced high-school level. The CosmoAmautas book ${ }^{7}$ is a unique open-access resource for teachers who want to integrate astrophysics in their classes, combining the theoretical framework with our enquiry-based learning activities.

\section{Societal objectives beyond STEM} Capitalizing on astronomy's potential to create awareness of climate change ${ }^{8}$, our curriculum on exoplanets gave us the opportunity to discuss the notion that there is no alternative planet ' $\mathrm{B}$ ' for humans to live on. The discussion on the local causes and impact of the climate crisis in Peru was led by a female Peruvian environmental engineer, focusing on the importance of the protection of the Amazon rainforest (12\% of which is in Peruvian territory).

In line with our objectives of equality, diversity and inclusion, we aimed to provide teachers and students with a diverse set of science role models, whom they can relate to. We thus designed our team to be at 
least $50 \%$ female, and we paid particular attention to gender balance and cultural and ethnic diversity in the selection of the participants, with $35 \%$ of the teachers identifying Indigenous languages (Quechua, Aymara, Cocama, Shawi, Shipibo-Conibo, Yagua and Yanesha) as their mother tongue. An objective of the following years is to translate the CosmoAmautas book to a few of these languages. A lecture on ancestral astronomy during the Inca Empire opened the workshop. The awareness of the relevant role astronomy played for our ancestors clearly increased the enthusiasm and identification of the teachers. In collaboration with designers and specialized historians, schematics of the Inca dark constellations have been developed and will be soon available in Stellarium (open-source software for planetaria) and on our webpage (Fig. 1). Interactive lectures and discussions on strategies to ensure gender balance in the school classroom were included, following UNESCO guidelines ${ }^{9}$, as well as a workshop on growth mindset. The response of the teachers was very positive and committed. Many expressed that they were previously unaware of possible unconscious biases. After the discussions, a few of them who had started astronomy clubs in their schools noticed that they had indeed received a much higher enrolment from male students. As a consequence of the discussion, they decided to actively encourage more girls to join them, achieving more gender-balanced clubs.

\section{Outcomes and impact assessment}

All the teachers who participated in an anonymous feedback survey reported that the workshop either met or exceeded their expectations, with $95 \%$ of the respondents having learnt not only new scientific concepts but also techniques for improving their online teaching methods. In addition to reproducing the activities from our curriculum, they also created and developed their own hands-on activities in groups. In this way, they honed their skills to implement the enquiry-based learning methodology in other school subjects beyond astronomy, which is the plan for $93 \%$ of the teachers.

The most direct evidence of the impact of the programme is the implementation of after-school astronomy clubs, AstroClubs, which began to take shape out of the teachers' own drive, even before the workshop sessions had culminated. In these 14 AstroClubs, more than 260 motivated students from rural high schools, aged 10 to 16 , are currently exploring astrophysics topics through enquiry-based learning activities together with their teachers. When asked for their motivation, the students reported joining the clubs to "go to Mars", "look for aliens" and "explore space with a telescope". To support their enthusiasm, CosmoAmautas has equipped each one of these AstroClubs with a telescope and 'rural' sky observation kits for all, which consist of planispheres and compasses to locate planets and constellations with the naked eye, without the need for a cell phone or other technologies. We will measure the success of the programme as it progresses by assessing the number of students from the AstroClubs who participate in national science events such as national science fairs, astrophysics Olympiads and similar events, where rural participation is usually limited. Early indications of positive impact have been already observed, with AstroClubs from Loreto and Moquegua participating in an international measurement of Earth's diameter comprising 105 countries (Eratosthenes project, https://eratosthenes. ea.gr). This was the first time that Peruvian schools from outside the capital were represented at this event.

Over the next few years, CosmoAmautas aims to offer the teacher training workshop and to open new AstroClubs in more regions in Peru, setting a precedent for other countries with limited scientific infrastructure. At the same time, the programme intends to showcase the intrinsic interest of the younger Peruvian generations for basic sciences such as astronomy. Our goal is to motivate local policymakers to redirect more funding into the research in these fields, shifting the current paradigm of local universities away from only training professionals towards becoming producers of new knowledge. Indeed, the research and development budget in Peru going into STEM comprises $0.12 \%$ of its gross domestic product, which is among the lowest in the Americas $(0.36 \%$ in Chile, $1.27 \%$ in Brazil and $2.8 \%$ in the United States $)^{10}$. In the long term, we aspire to promote and decentralize the technological and scientific development of the country, while aiming for a world where future generations not only look up at the same sky but also have equitable access to the resources required to explore it.

Gabriela Calistro Rivera (D) $1 \times$, Daniella Bardalez Gagliuffi², Diego Alvarado Urrunaga ${ }^{3}$, Lisseth Gonzales Quevedo ${ }^{4}$,
Daniel Kleffman ${ }^{5}$, Erick Meza ${ }^{6,7}$,

Adita Quispe Quispe ${ }^{8}$,

Jenny Margot Ramos Lázaro4,

José Ricra ${ }^{9}$, Bruno Rodríguez Marquina ${ }^{10}$ and

Erika Torre Ramirez (D) 3

${ }^{1}$ European Southern Observatory (ESO), Garching

bei München, Germany. ${ }^{2}$ American Museum of Natural History, New York, NY, USA. ${ }^{3}$ Universidad Nacional de Ingeniería, Lima, Peru. ${ }^{4}$ Universidad Nacional Mayor de San Marcos, Lima, Peru. ${ }^{5}$ Breda University of Applied Sciences, Breda, The Netherlands. ${ }^{6}$ Comisión Nacional de Investigación y Desarrollo Aeroespacial del Perú - CONIDA, Lima, Peru. ${ }^{7}$ Observatorio Astronómico de Moquegua, Moquegua, Peru. ${ }^{8}$ Instituto Geofísico Del Perú (IGP), Lima, Peru. ${ }^{9}$ Observatorio Astronómico AFARI, Tarma, Peru. ${ }^{10}$ Pontificia Universidad Católica del Perú (PUCP), Lima, Peru.

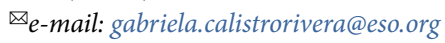

Published online: 18 February 2022

https://doi.org/10.1038/s41550-022-01605-2

References

1. González-García, A. et al. Lat. Am. Antiq. 32, 154-172 (2021).

2. Aksaker, N. et al. Mon. Not. R. Astron. Soc 493 1204-1216 (2020).

3. The 17 Goals (United Nations, 2015); https://sdgs.un.org/goals

4. Acting Now to Protect the Human Capital of Our Children: The Costs of and Response to COVID-19 Pandemic's Impact on the Education Sector in Latin America and the Caribbean (World Bank, 2021); https://openknowledge.worldbank.org/ handle/10986/35276

5. PISA 2018 Results (Volume II): Where All Students Can Succeed (OECD, 2019); https://doi.org/10.1787/b5fdlb8f-en

6. Peru statistics database. (INEI, 2020); https://www.inei.gob.pe/ estadisticas/indice-tematico/analfabetismo-y-alfabetismo-8036

7. Calistro Rivera, G. et al. Preprint at https://arxiv.org/abs/2109. 11945 (2021).

8. Anderson, A. \& Maffey, G. Nat. Astron 5, 861-863 (2021).

9. Cracking the Code: Girls' and Women's Education in Science, Technology, Engineering and Mathematics (STEM) (UNESCO, 2017); https://unesdoc.unesco.org/ark:/48223/pf0000253479 10. Bajak, A. Nature 576, S65-S67 (2019).

\section{Acknowledgements}

CosmoAmautas is possible thanks to the support of the International Astronomical Union (IAU) Office of Astronomy for Development, which is a joint project of the IAU and the South African National Research Foundation (NRF) with the support of the Department of Science and Innovation (DSI). We thank the Open Astronomy Schools programme for the donation of ten telescopes, which are currently being used in the after-school AstroClubs. We thank ESO, ESA and CONIDA for their generous donation of astronomy-related material, such as posters, stickers, booklets and telescope models. This Comment was written on behalf of the CosmoAmautas team, whose members and collaborators include (in addition to the co-authors) S. Casas, E. Calcina, V. Navarrete, A. Verán, N. Málaga and M. Zeballos. We thank A. Verán and M. Kaasinen for content advice and proofreading. G.C.R. acknowledges the ESO Fellowship Program and a Gruber Foundation Fellowship grant sponsored by the Gruber Foundation and the International Astronomical Union.

\section{Competing interests}

The authors declare no competing interests. 